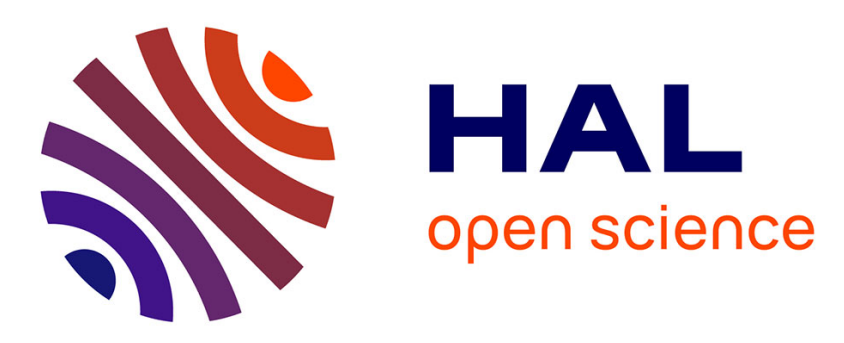

\title{
Structure-function strategies to improve the pharmacological value of animal toxins.
}

\author{
Michel de Waard, Jean-Marc Sabatier
}

\section{To cite this version:}

Michel de Waard, Jean-Marc Sabatier. Structure-function strategies to improve the pharmacological value of animal toxins.. Abba J. Kastin. Peptides - Handbook of Biologically Active Peptides, Elsevier, pp.415-419, 2006, 10.1016/B978-012369442-3/50062-3 . inserm-00394127

\section{HAL Id: inserm-00394127 https://www.hal.inserm.fr/inserm-00394127}

Submitted on 10 Jun 2009

HAL is a multi-disciplinary open access archive for the deposit and dissemination of scientific research documents, whether they are published or not. The documents may come from teaching and research institutions in France or abroad, or from public or private research centers.
L'archive ouverte pluridisciplinaire HAL, est destinée au dépôt et à la diffusion de documents scientifiques de niveau recherche, publiés ou non, émanant des établissements d'enseignement et de recherche français ou étrangers, des laboratoires publics ou privés. 


\section{Structure-function strategies to improve the}

\section{pharmacological value of animal toxins}

Michel De Waarda and Jean-Marc Sabatier,c,**

${ }^{a}$ Laboratoire Canaux Calciques, Fonctions et Pathologies, Inserm U607, CEA, DRDC, 17 Rue des Martyrs, 38054 Grenoble Cedex 09, France

b Laboratoire d'Ingénierie des Protéines, CNRS FRE 2738, Boulevard Pierre Dramard, 13916 Marseille Cedex 20, France

c Laboratoire CellPep S.A., 13-15 Rue Ledru-Rollin, 13015 Marseille, France

${ }^{*}$ Corresponding author.

Tel.: +334916988 52; fax: +33491657595

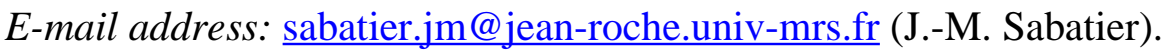




\begin{abstract}
Animal venoms are rich sources of bioactive compounds that possess obvious pharmacological, therapeutic, and/or biotechnological values. A majority of these compounds are peptides that mainly target enzymes, membrane receptors, or ion channels. These peptides are most often in a size range that allows their production in vitro by chemical synthesis or genetic engineering. Unfortunately, they rarely display the required characteristics in terms of selectivity, affinity, stability and targeting with regard to the desired application. In recent years, a number of structural approaches or strategies have been developed to improve the intrinsic potential of venom peptides. They are reviewed herein for their effectiveness.
\end{abstract}

\title{
1. Chemical synthesis versus genetic engineering - Pros and cons
}

In animal venoms, peptide sizes are mainly in the range of 0.4 to $8.0 \mathrm{kDa}$. This would correspond to peptides of ca. 4 to 70 amino acid residues. Because of the intrinsic structural complexities of these peptides (different types of fold and 1-5 disulfide bridges), this size range implicates that only a fraction of them $(<50$-mer peptides $)$ can easily be produced by chemical synthesis [17,22]. Longer peptides are more difficult to produce chemically and have generally required the use of molecular biology techniques for their productions. Basically, chemical synthesis has several advantages over genetic engineering, making the former approach more appropriate to improve the structural and functional properties of peptides of interest. In particular, the possibility to incorporate non-natural amino acid derivatives or to form non-amide pseudo-peptide bonds is a definite benefit over genetic production. Also, the high yields of peptide productions are assets for detailed structural analyses and functional characterizations of the peptides. Finally, the production of peptides for therapeutic purposes is less cumbersome when a chemical approach is chosen over a genetic one. Indeed, safety, reproducibility, and lack of cell contaminants clearly argue in 
favor of chemical synthesis. In fact, the vast majority of studies relating structure-function analyses are based on peptides produced by chemical synthesis.

\section{Peptide improvement requires solid basic knowledge on the lead peptide properties}

For valuable strategies of peptide improvement, one needs to know the peptide target(s), 3-

D structure, functional effect(s), and specific application(s) pursued. With uncharacterized venom peptides, identification of actual target(s) is generally a difficult task. Pharmacological profiling using well defined screening approaches often reveals that peptides recognize several targets (e.g., different ion channel types) or may be derived for multiple applications. From pharmacological studies, useful information can be gained that includes peptide affinity for its target(s), selectivity profile, and mode of action. Gathering structural data is an essential step in defining the interacting surfaces of both the peptide and its target(s). In most cases, venom peptides are readily amenable to ${ }^{1} \mathrm{H}-\mathrm{NMR}$-based 3-D structure determination. Alternatively, computer-assisted molecular modeling is an interesting route that can be used for peptide structural analysis provided that appropriate 3D structures of related compounds are available from databases to serve as templates. The accurate structural determination of the peptide's target(s) or, at least, of its binding site(s) is a considerably more difficult challenge. Although this condition is not always met satisfactorily, it is clearly beneficial for the design of novel and more potent peptide analogs. Structural information, from both peptide and target, allow more sophisticated analyses that include in silico molecular docking simulations. From the most energetically favorable docking solutions, relevant hypotheses can be drawn on the characteristics of the interacting surfaces involved. These can then be verified experimentally by using complementary mutagenesis of the peptide and its target(s), leading to a precise view of the peptide's structural and functional properties [11]. 


\section{Design of novel peptide analogs by selective residue substitution(s)}

This method is largely employed to obtain novel analogs with unique pharmacological properties, or to pursue detailed structure-function studies. It represents the method of choice to address whether or not certain amino acid residues from the peptide are involved in target recognition and binding. It also allows a precise mapping of the peptide interacting surface, provided that the substituted residues do not alter the global peptide conformation. Generally, residue substitutions are made on a systematic basis, using the alanine-scanning approach. By this method, the contribution of scorpion toxin functional dyads to $\mathrm{K}^{+}$channel blockage was experimentally investigated [4,18]. Mutation of amino acid residues also represents a powerful strategy to improve the pharmacological value and therapeutic potential of a particular peptide. Two general strategies can be envisioned. First, substituting amino acid residues within the peptide active site. This strategy can be used to increase the affinity of the peptide towards one specific target, or for improving its selectivity profile (similar or increased affinity for one target, but decreased affinity towards one or several other targets) [23], or both [1]. Second, substituting amino acid residues outside the peptide active site. This strategy may generate more subtle variations in peptide pharmacological properties, although in a more unpredictable manner. Indeed, it is expected that amino acid residue substitutions outside the active site should slightly alter the peptide's interacting surface. Stronger conformational changes are expected from substitutions that target residues key for a proper peptide folding. For instance, it has been shown that single residue mutations in scorpion maurotoxin can affect its pattern of disulfide bridging and, hence, its conformation and pharmacology [9]. Apart from acting on peptide affinity and selectivity by substituting natural amino acid residues by others, one can also act on peptide stability (e.g., sensitivity to proteases) by using non-natural residues for the substitutions instead. It is worth mentioning, however, that a similar protease resistance can also be acquired by selective substitutions based on natural amino acid residues only. Owing to the 
conformational change of the peptide that is potentially induced by residue substitution, it is wise to gain insight of the structural features of each analog designed, produced and tested for bioactivity (e.g., 3-D solution structure determination by ${ }^{1} \mathrm{H}-\mathrm{NMR}$, circular dichroïsm analysis, molecular modeling). Combined with docking simulation experiments of these analogs over their targets, all the data gathered should be of value for a still more rational design of novel more potent and/or selective peptide analogs by an iterative feedback information process.

\section{Benefits of size reduction in peptides}

Since many large peptides (> 50-mer) are not readily amenable to chemical production, it may appear attractive to reduce their size without compromising on their pharmacological value. Unfortunately, this strategy has seldom been used and too little information is available from the literature to reasonably draw a conclusion about their effectiveness. However, some remarks may be pertinent. Size reduction of peptides may be used for delineation of their pharmacophore(s). Similarly, it may prove useful for restricting the peptide selectivity profiles. This approach would certainly be beneficial in terms of production costs and should bypass the complex use of a genetic production strategy. In the case of venom peptides, one would expect that such a strategy should severely decrease the pharmacological potencies of the resulting low mass analogs since the integrity of the various toxin folds characterized so far, that would presumably be affected by peptide size reduction, is required for the correct spatial distribution of functionally key amino acid residues [17]. However, this strategy might be particularly valuable for peptides presenting small pharmacophores, and thus less susceptible to alteration of the active site conformation by peptide size trimming.

\section{Relevance of chimera and labeling approaches}


Structure-activity relationship studies have demonstrated that a given venom peptide can be active on several targets (large selectivity profile) through different interacting surfaces. The existence of a variety of folds in venom peptides [17] allows the occurrence of distinct relative localizations of the active interacting surfaces [21]. For instance, it has been documented that scorpion toxins generally fold according to the $\alpha / \beta$ architectural motif (an $\mathrm{N}$-terminal helical structure connected by disulfide bridges to a C-terminal antiparallel, twoor three-stranded, $\beta$-sheet structure) [20]. Many of these scorpion venom peptides act on voltage-gated $\mathrm{K}^{+}(\mathrm{Kv})$ channels through interacting surfaces primarily involving their $\beta$ sheet structures, whereas they may interact with small conductance $\mathrm{Ca}^{2+}$-activated $\mathrm{K}^{+}(\mathrm{SK})$ channels through opposite interacting surfaces that implicate their helical structures. Given the fact that interacting surfaces of some venom peptides (with enlarged target specificity) are associated with residues of specific secondary structures, it may be worth using a chimera approach to produce peptide analogs with either enlarged or restricted pharmacological profiles. A representative example of such an approach was provided with a chimera made by the $\mathrm{N}$-terminal helical region of maurotoxin and the $\mathrm{C}$-terminal $\beta$-sheet region of HsTx1 [19]. In this case, a "gain of function" was obtained since the chimera acquires the activity of maurotoxin on SK channels while preserving the pharmacological profile of HsTx1 on Kv channels. Conversely, a "loss of function" can be attributed to this chimera since the activity of maurotoxin on a specific Kv channel subtype is lost. The cited approaches relate to change in peptide selectivity (enlargement or restriction). Of note, the chimera approach can tentatively be used to modulate peptide affinity towards a particular target without altering the initial pharmacological profile. This was successfully experienced using a chimera of butantoxin and maurotoxin in which the addition of the $9 \mathrm{~N}$-terminal residues of butantoxin to the $31 \mathrm{C}$-terminal residues of maurotoxin provides some additional molecular contacts for the interaction with Kv1.2 channel (pharmacophore enlargement), 
thereby enhancing peptide affinity for this channel subtype [15].

Other technical applications are worth mentioning. First, peptides can be modified for tracking purposes. Tracking can be useful for the determination of peptide tissue distribution, sub-cellular receptor localization, or classical binding experiments. For example, such an "add-on" function has proven valuable for demonstrating the cellpenetration ability of maurocalcine, a venom peptide acting on intracellular ryanodinesensitive $\mathrm{Ca}^{2+}$ channels [7]. Site-directed chemical modifications of peptides, by labeling with fluorescent derivatives or radioactive materials (e.g., ${ }^{125}$ I), or biotinylation, are routinely used for binding experiments. Second, venom peptides can be derived to become multifunctional. Chemical coupling to other molecules, such as antibodies or independent functional domains, may represent an interesting strategy for novel types of "gain of function", including cell targeting, cell toxicity, cell labeling, cell delivery of compounds, etc. Unfortunately, these research avenues are still largely unexploited and their add-on values under-evaluated.

\section{Strategies implying a change in the pattern of half-cystine pairs}

Venom peptides are often highly reticulated structures. It has been evidenced that disulfide bridges contribute to the acquisition and stability of the various peptide folds. Secondary structures are frequently connected to each other by at least one disulfide bridge. The pattern of a half-cystine connection is also crucial for the correct spatial distribution of the functionally key amino acid residues from the peptide interacting surfaces. Strategies based on the pattern of half-cystine pairs can be classified into three categories. The first one aims at increasing the number of disulfide bridges. The extra half-cystine pair might decrease peptide flexibility and, possibly, that of the interacting surfaces, provided that the additional disulfide bridge is inserted in an appropriate location within the peptide amino acid sequence. In turn, a reduced flexibility may lessen peptide adaptability to their target(s) and 
one may expect a greater pharmacological selectivity for the analogs designed this way. However, an experimental validation of this approach is still needed. In contrast, the second strategy relies on a reduction in the number of peptide half-cystine pairs instead; a strategy that may conceivably increase peptide flexibility thereby potentially affecting its pharmacological behavior. In both cases, adding or removing a disulfide bridge may have consequences for the pattern of other half-cystine pairs, therefore highlighting the need for a careful structural characterization of the analogs. The third strategy relies on a change in the pattern of disulfide bridges within the peptide (with or without variation in the number of half-cystine pairs). Enforcing new disulfide bridge arrangements in a peptide is an effective way to alter the conformation of its pharmacophore(s) and, hence, its pharmacology. This strategy has been applied twice for the scorpion maurotoxin. By replacing two half-cystine residues belonging to two different disulfide bridges, a novel three-disulfide-bridged maurotoxin analog was produced in which the third half-cystine pair was unique. This analog still folded along the common $\alpha / \beta$ architectural motif of scorpion toxins, while presenting a new selectivity towards Kv channels [8]. Also, by using an innovative strategy of chemical synthesis, a four-disulfide-bridged maurotoxin variant was produced in which the two last bridges were differently arranged as compared with native maurotoxin (although half-cystine pairings are similar to those found in other toxins from the same structural family). Again, for this analog, the common architectural motif was maintained, but the change in disulfide bridge pattern was accompanied by changes in pharmacology [16].

It is worth mentioning that removal of all half-cystine pairs from a reticulated venom peptide - in the cases where it has been studied - abolishes peptide bioactivity, suggesting that the presence of all disulfide bridges are required for the maintenance of peptide fold. This property has been taken into consideration for peptide-based immunizations during vaccination programs, with the aim of neutralizing the toxicity of properly folded and 
reticulated toxins $[10,24]$.

\section{What about the dipole moment of venom peptides?}

Many venom peptides exhibit a marked electric charge anisotropy. A formal representation of this anisotropy is given by the peptide dipole moment. It has been proposed that the dipole moment guides and orients the peptide towards its target(s), thereby being key for peptide specificity [3]. Although the concept is attractive by itself, it remains to be supported experimentally. In fact, the main flaw of this concept is that it is somehow incompatible with the existence of several interacting surfaces, which are sometimes located on opposite faces of the peptide (e.g. activity of maurotoxin on both SK and $\mathrm{Kv}$ channels involving opposite faces). Noteworthy, the noticeable charge anisotropy of venom peptide maurocalcine, which possesses a highly basic face, is likely to contribute to its singular ability to translocate into cells where the peptide acts [7]. This property is shared with other cell-penetrating, structurally-unrelated, peptides such as Tat and penetratin [13].

\section{Functional derivation of venom peptides: a search for new applications}

Venom peptides exhibit a great variety of folds. In the case of animal toxins acting on ion channels, up to 14 different types of fold were identified [17]. Along with other favorable characteristics, such as small size and high stability, diversity of fold can advantageously be used for a functional derivation of venom peptides. Indeed, active sites of venom peptides can be substituted by functionally unrelated pharmacophores of biological value to benefit of the gainful peptide characteristics. For example, the CD4 binding surface for the HIV-1 gp120 envelope glycoprotein has been successfully transferred into the structure of a scorpion toxin possessing the $\alpha / \beta$ scaffold [12]. This approach leads to the production of interesting anti-HIV compounds with demonstrated efficacies in vitro. Venom peptides with 
novel pharmacophores represent new tools for biological, biotechnological and medical applications. A therapeutic development may also be envisioned in case the novel compounds possess the appropriate drug properties.

Other types of functional derivation can be achieved that are not necessarily based on the replacement of the peptide pharmacophore by an exogenous one. In that case, different properties of the peptide are derived, such as cell targeting, receptor recognition, membrane translocation, nucleic acid binding, etc. It is worth noting that, in some cases, it is mandatory to eliminate the original pharmacological activity of the peptide to prevent some unwanted side effects. For instance, the pharmacological activity of maurocalcine on intracellular ryanodine-sensitive calcium channels can be suppressed [6] in order to use only its cellpenetration property for the cell-entry of larger non-membrane permeable compounds, including whole proteins [7]. In other cases, structures of venom peptides can be modified to properly target toxins to a given cell type in vivo. Such a strategy has been developed in the case of immunotoxins, in which a toxin is coupled to a cell-targeting ligand or antibody. The main focus of the immunotoxin approach is targeted cancer therapy [14]. So far, bacterial and plant toxins have been used, but the technique could prove valuable with animal toxins.

\section{Improving peptide stability}

Since the ever-increasing interest in studying animal venom peptides is related to their potential use in therapeutics, it appears suitable to optimize their stability in vivo. Several methods can be used to improve peptide stability. Among them, one can mention: (i) amino acid residue substitution(s) aimed at protecting the peptide from proteolytic cleavage. Residue replacement(s), which should not alter the pharmacophore properties, can be achieved using L-, D-, or non-natural amino acid residues (including residues modified on their side-chains). (ii) A selective chemical modification of the peptide (e.g. changing the nature of the $\mathrm{N}$ - (acetylation) [5] and/or C-terminal extremities (amidation, addition of a 
specific group such as a fatty acid). Such a strategy may also be valuable for improving the pharmacological characteristics of a given peptide, as shown in the case of ShK sea anemone toxin in which grafting of a phosphotyrosine moiety at the N-terminus enhances its selectivity profile towards the Kv1.3 potassium channel [2]. (iii) The replacement of natural peptide bonds by pseudo-peptide bonds during chemical synthesis (e.g., $\mathrm{CO}-\mathrm{NH}$ by NH-CO, or $\mathrm{CH}_{2}-\mathrm{CH}_{2}$, or $\mathrm{CO}-\mathrm{CH}_{2}$, etc). (iv) Cysteine-based cyclization of the peptide. These modifications, which might, by themselves, be detrimental to peptide biological properties, should be attempted only to overcome specific problems, such as short peptide half-life, poor peptide stability and/or solubility, and inadequate tissue distribution. Of note, although peptide modifications have been used in the development of several therapeutically useful peptides, it has seldom been put into application for venom peptides. Apart from chemical modifications of the peptides, it is also possible to act on peptide stability by using specific delivery systems (liposomes, nanoparticles, glycan polymers, hydrogels). The latter do not belong to structure-function strategies and thus will not be developed further.

\section{Concluding remarks}

Despite the richness in biologically active venom peptides, they rarely come with the desired set of characteristics. A rationale structure-function strategy is therefore a key step to obtain a valuable compound for any given application. Strategies are now numerous and have proven to be efficient to reach these goals. The choice of a particular strategy actually depends on the peptide biological properties that one seeks to reinforce, create, or suppress. Not all strategies are equivalent and, sometimes, a combination thereof should be used. An iterative feedback information process is often required, which combines the benefits of several approaches, to generate compounds with the appropriate set of requisite characteristics. Combined strategies will prove valuable for the future development of 
venom peptide-derived drugs in the treatment of specific human diseases.

\section{References}

[1] Alessandri-Haber N, Lecoq A, Gasparini S, Grangier-Macmath G, Jacquet G, Harvey $\mathrm{AL}$, et al. Mapping the functional anatomy of $\mathrm{BgK}$ on $\mathrm{Kv} 1.1, \mathrm{Kv} 1.2$, and $\mathrm{Kv} 1.3$. Clues to design analogs with enhanced selectivity. J Biol Chem 1999;274:35653-61.

[2] Beeton C, Pennington MW, Wulff H, Singh S, Nugent D, Crossley G, et al. Targeting effector memory $\mathrm{T}$ cells with a selective peptide inhibitor of Kv1.3 channels for therapy of autoimmune diseases. Mol Pharmacol 2005;67:1369-81.

[3] Blanc E, Sabatier JM, Kharrat R, Meunier S, El Ayeb M, Van Rietschoten J, et al. Solution structure of maurotoxin, a scorpion toxin from scorpio maurus, with high affinity for voltage-gated potassium channels. Proteins 1997;29:321-33.

[4] Dauplais M, Lecoq A, Song J, Cotton J, Jamin N, Gilquin B, et al. On the convergent evolution of animal toxins. Conservation of a diad of functional residues in potassium channel-blocking toxins with unrelated structures. J Biol Chem 1997;272:4302-9.

[5] de Haan EC, Wauben MH, Wagenaar-Hilbers JP, Grosfeld-Stulemeyer MC, Rijkers DT, Moret EE, et al. Stabilization of peptide guinea pig myelin basic protein $72-85$ by N-terminal acetylation - Implications for immunological studies. Mol Immunol 2004;40:943-8. 
[6] Estève E, Smida-Rezgui S, Sarkozi S, Szegedi C, Regaya I, Chen L, et al. Critical amino acid residues determine the binding affinity and the $\mathrm{Ca}^{2+}$ release efficacy of maurocalcine in skeletal muscle cells. J Biol Chem 2003 ;278:37822-31.

[7] Estève E, Mabrouk K, Dupuis A, Smida-Rezgui S, Altafaj X, Grunwald D, et al. Transduction of the scorpion toxin maurocalcine into cells - Evidence that the toxin crosses the plasma membrane. J Biol Chem 2005;280:12833-9.

[8] Fajloun Z, Ferrat G, Carlier E, Fathallah M, Lecomte C, Sandoz G, et al. Synthesis, ${ }^{1} \mathrm{H}$ NMR structure, and activity of a three-disulfide-bridged maurotoxin analog designed to restore the consensus motif of scorpion toxins. J Biol Chem 2000;275;13605-12.

[9] Fajloun Z, Mosbah A, Carlier E, Mansuelle P, Sandoz G, Fathallah M, et al. Maurotoxin Versus Pi1/HsTx1 toxins: Toward new insights in the understanding of their distinct disulfide bridge patterns. J Biol Chem 2000;275:39394-402.

[10] Gazarian KG, Gazarian T, Hernández R, Possani LD. Immunology of scorpion toxins and perspectives for generation of anti-venom vaccines. Vaccine 2005;23:3357-68.

[11] Gilquin B, Racape J, Wrisch A, Visan V, Lecoq A, Grissmer S, et al. Structure of the BgK-Kv1.1 complex based on distance restraints identified by double mutant cycles. Molecular basis for convergent evolution of Kv1 channel blockers. J Biol Chem 2002;277:37406-13.

[12] Huang CC, Stricher F, Martin L, Decker JM, Majeed S, Barthe P, et al. Scorpion-toxin mimics of CD4 in complex with human immunodeficiency virus gp120 crystal 
structures, molecular mimicry, and neutralization breadth. Structure 2005;13:755-68.

[13] Lindgren M, Hallbrink M, Prochiantz A, Langel U. Cell-penetrating peptides. Trends Pharmacol Sci 2000;21:99-103.

[14] MacDonald GC, Glover N. Effective tumor targeting: strategies for the delivery of armed antibodies. Curr Opin Drug Discov Devel 2005;8:177-83.

[15] M'Barek S, Chagot B, Andreotti N, Visan V, Mansuelle P, Grissmer S, et al. Increasing the molecular contacts between maurotoxin and Kv1.2 channel augments ligand affinity. Proteins 2005; (in press).

[16] M'Barek S, Lopez-Gonzalez I, di Luccio E, Visan V, Grissmer S, Judge S, et al. A maurotoxin with constrained standard disulfide bridging - Innovative strategy of chemical synthesis, pharmacology, and docking on $\mathrm{K}^{+}$channels. J Biol Chem 2003;278:31095-104.

[17] Mouhat S, Jouirou B, Mosbah A, De Waard M, Sabatier JM. Diversity of folds in animal toxins acting on ion channels. Biochem J 2004;378:717-26.

[18] Mouhat S, De Waard M, Sabatier JM. Contribution of the functional dyad of animal toxins acting on voltage-gated Kv1-type channels. J Pep Sci 2005;11:65-8.

[19] Regaya I, Beeton C, Ferrat G, Andreotti N, Darbon H, De Waard M, et al. Evidence for domain-specific recognition of SK and Kv channels by MTX and HsTx1 scorpion toxins. J Biol Chem 2004;279:55690-6. 
[20] Rodríguez de la Vega RC, Merino E, Becerril B, Possani LD. Novel interactions between $\mathrm{K}^{+}$channels and scorpion toxins. Trends Pharmacol Sci 2003;24:222-7.

[21] Rodríguez de la Vega RC, Possani LD. Current views on scorpion toxins specific for $\mathrm{K}^{+}$-channels. Toxicon 2004;43:865-75.

[22] Sabatier JM. Chemical synthesis and characterization of small proteins: example of scorpion toxins. In: Rochat H, Martin-Eauclaire MF, editors. Animal toxins. Basel, Switzerland: Birkhaüser Verlag; 2000. p. 196-216.

[23] Shakkottai VG, Regaya I, Wulff H, Fajloun Z, Tomita H, Fathallah M, et al. Design and characterization of a highly selective peptide inhibitor of the small conductance calcium-activated K+ channel, SKCa2. J Biol Chem 2001;276:43145-51.

[24] Zenouaki I, Kharrat R, Sabatier JM, Devaux C, Karoui H, Van Rietschoten J, et al. In vivo protection against Androctonus australis hector scorpion toxin and venom by immunization with a synthetic analog of toxin II. Vaccine 1997;15:187-94. 